\title{
Use and subjective experience of the impact of a motor-assisted movement exerciser in people with amyotrophic lateral sclerosis: a multicentre observational study
}

\section{André Maier ( $\square$ andre.maier@charite.de )}

Centre for ALS and other Motor Neuron Disorders, Charité - Universitätsmedizin Berlin, Corporate Member of Freie Universität Berlin und Humboldt-Universität zu Berlin

Marcel Gaudlitz

Ambulanzpartner Soziotechnologie APST GmbH, Berlin

Torsten Grehl

Alfried Krupp Krankenhaus, Department of Neurology, Centre for ALS and Other Motor Neuron Disorders, Essen

\section{Ute Weyen}

Berufsgenossenschaftliches Universitätsklinikum Bergmannsheil, Centre for ALS and Other Motor Neuron Disorders, Bochum

\section{Robert Steinbach}

Jena University Hospital, Hans Berger Department of Neurology, Jena

Julian Großkreutz

University of Lübeck, Precision Neurology, Lübeck

\section{Annekatrin Rödiger}

Jena University Hospital, Hans Berger Department of Neurology, Jena

\section{Jan Christoph Koch}

Universitätsmedizin Göttingen, Department of Neurology, Göttingen

\section{Teresa Lengenfeld}

Universitätsmedizin Göttingen, Department of Neurology, Göttingen

\section{Patrick Weydt}

Universitätsklinikum Bonn - Klinik für Neurodegenerative Erkrankungen, Bonn

René Günther

Technische Universität Dresden, Department of Neurology, Dresden

Joachim Wolf

Diakonissenkrankenhaus Mannheim, Department of Neurology, Mannheim

\section{Petra Baum}

Universitätsklinikum Leipzig, Department of Neurology, Leipzig

\section{Moritz Metelmann}


Universitätsklinikum Leipzig, Department of Neurology, Leipzig

Johannes Dorst

University of Ulm, Department of Neurology, Ulm

Albert Christian Ludolph

University of Ulm, Department of Neurology, Ulm

\section{Dagmar Kettemann}

Centre for ALS and other Motor Neuron Disorders, Charité - Universitätsmedizin Berlin, Corporate Member of Freie Universität Berlin und Humboldt-Universität zu Berlin

\section{Jenny Norden}

Centre for ALS and other Motor Neuron Disorders, Charité - Universitätsmedizin Berlin, Corporate Member of Freie Universität Berlin und Humboldt-Universität zu Berlin

\section{Ruhan Yasemin Koc}

Centre for ALS and other Motor Neuron Disorders, Charité - Universitätsmedizin Berlin, Corporate Member of Freie Universität Berlin und Humboldt-Universität zu Berlin

\section{Bertram Walter}

Centre for ALS and other Motor Neuron Disorders, Charité - Universitätsmedizin Berlin, Corporate Member of Freie Universität Berlin und Humboldt-Universität zu Berlin

\section{Barbara Hildebrandt}

Ambulanzpartner Soziotechnologie APST GmbH, Berlin

\section{Christoph Münch}

Ambulanzpartner Soziotechnologie APST GmbH, Berlin

\section{Thomas Meyer}

Centre for ALS and other Motor Neuron Disorders, Charité - Universitätsmedizin Berlin, Corporate Member of Freie Universität Berlin und Humboldt-Universität zu Berlin

\section{Susanne Spittel}

Ambulanzpartner Soziotechnologie APST GmbH, Berlin

\section{Research Article}

Keywords: Amyotrophic lateral sclerosis, motor-assisted movement exerciser, device-assisted physical therapy, user experiences

Posted Date: January 31st, 2022

DOI: https://doi.org/10.21203/rs.3.rs-1254494/v1

License: (c) (i) This work is licensed under a Creative Commons Attribution 4.0 International License. Read Full License 


\section{Abstract}

Objective: Motor-assisted movement exercisers (MME) enable device-assisted physical therapy delivered in domestic settings. This observational study captures the frequency of MME use, subjective experience of the therapy provided and MME recommendation.

Method: The prospective cohort study was implemented at ten ALS centres (02/2019-10/2020) and coordinated by the research platform Ambulanzpartner. Participants assessed symptom severity, frequency of use and subjective benefit of therapy on a numerical rating scale (NRS, 0 to 10 points). The likelihood of recommending the MME was determined by the Net Promotor Score (NPS).

Results: Data for 144 participants were analysed. Weekly MME applications ranged from 1 to 4 in $41 \%, 5$ to 7 in $42 \%$ and $>7$ in $17 \%$ of participants. Particularly positive results were achieved in the following domains: amplification of a sense of achievement (67\%), diminution of the feeling of having rigid limbs $(63 \%)$, diminution of the feeling of being immobile (61\%), improvement of general wellbeing $(55 \%)$ and reduction of muscle stiffness (52\%). Participants with more pronounced self-rated muscle weakness were more likely to note a beneficial effect on preservation and improvement of muscle strength under MME treatment $(p<0.05)$. The NPS for the MME was high $(+61)$.

Conclusion: High-frequency MME-assisted treatment (defined as a minimum of five sessions a week) was administered in the majority of participants (59\%) in addition to physical therapy. Most patients stated to have achieved their individual objectives of MME intervention as evidenced by a high level of satisfaction. The results underline the justification for extended MME treatment as part of the ALS care concept.

\section{Introduction}

Amyotrophic lateral sclerosis (ALS) is a fatal neurodegenerative disease that affects motor neurons and therefore causes muscular atrophy and changes in muscle strength and tone. The most common disease presentation is limb onset which is found in about two-thirds of people living with ALS. Almost each one of them experiences muscle weakness and stiffness of the arms and legs as the disease progresses (1). While ALS is an inevitably progressive degenerative disease, the provision of assistive devices (2) and physiotherapy are recommended and well established measures (3). There is a variety of individual therapeutic approaches, from strengthening and stretching of muscles, regulation of muscle tone to pain management and prevention of falls (4). There are no uniform therapy regimens given the variability among ALS disease courses. Multiple trials recommend regular moderate muscle strengthening training and aerobic exercises at submaximal effort (3). In addition to the positive effects on physical functionality, preclinical studies have demonstrated neuroprotection and prolonged motor neuron survival (5).

In ALS, the benefits of a mobility routine in combination with low to moderate intensity cycling exercises have already been demonstrated (6). As motor-assisted movement exercisers (MME) have already proven 
beneficial in other neurological diseases (7-9) they have hence been integrated into the standard of ALS care. The training options provided by an MME encompass active (muscle power), passive (motor power) and assistive (muscle and motor power) training for both arms and legs. The perceived benefits are a strengthening of muscle groups, reduction of spasticity and support of the cardiovascular system $(7,8)$. In addition, an MME mobilises the entire musculoskeletal system including joints and tendons while eliciting sensory inputs which in turn enhances sensorimotor feedback $(10,11)$.

In Germany, people with ALS can be provided with assistive devices and technology, such as an MME, all of which can be used at home and complement physiotherapy. While this is part of standard care, the provision of prescription exercise devices is dependent on a patient's individual medical situation. To date, there have been no systematic studies on the utilisation and provision of the MME in ALS.

The aim of the present observational study, therefore, was to identify the provision and utilisation situation including the patients' attitude towards using an MME.

For this study, we established the following hypotheses: (I) There is a correlation between frequency of use of the MME and self-reported symptoms (muscle stiffness and muscle strength). (II) The higher the frequency of use, the more participants report benefits in their self-assessment. (III) There is a correlation between the recommendation of the MME and the frequency of use or other clinical or epidemiological data.

\section{Materials And Methods \\ Study design}

A prospective multicentre observational cohort study was conducted at 10 specialised ALS treatment centres in Germany between February 2019 and January 2021. The study is reported according to the STROBE criteria (12).

\section{Participants}

Subjects meeting the following criteria were included in this study: possible, probable or definitive diagnosis of ALS according to the revised El Escorial criteria (13), medical indication for domestic use of an MME, provision of an MME via the case management network Ambulanzpartner Soziotechnologie (APST) $(2,14-16)$ and provision of informed consent for participation in the study. Participants with other severe life-limiting diseases or with clinically significant cognitive impairment were not eligible for this study.

\section{Ethical approval and consent to participate}

The study is in accordance with relevant guidelines and regulations and has been approved by the Medical Ethics Committee of Charité - Universitätsmedizin Berlin, Germany under code EA1/219/15. 
Subjects were supplied with information on the study, both verbally and in writing. Informed consent was obtained from all subjects.

\section{Setting}

\section{Case management}

Prior to the study, all of the 10 contributing specialised ALS centres had already joined the APST multicentre case management network where the established routine process is as follows: ALS-trained neurologists define the medical indication for MME therapy to be embedded in the standard of ALS care. APST case managers then notify providers of a patient's need for this device. Upon delivery, participants are granted a four-week trial period, after which individual recommendations for a therapy regime are determined in close consultation with ALS-trained neurologists, physical therapists and providers of assistive devices. In Germany, the outcome of the trial period determines whether or not the costs incurred will ultimately be absorbed by a patient's health insurance company. Due to the non-interventional design of the present study, a general recommendation for MME use was issued while patients were not given a recommendation as to the specific exercise routine to be followed.

\section{Assessment and data capture}

Case report forms were issued to neurologists, study coordinators and study assistants for the purpose of assessing clinical and demographic data while both print and online questionnaires and structured interviews were realised via the digital research platform APST for the purpose of assessing patientreported outcomes.

\section{Variables}

\section{Demographic and clinical data}

The demographic and clinical data collected included gender, age at symptom onset, disease duration, type of ALS and the self-administered ALS Functional Rating Scale-revised (ALSFRS-R) (17). The ALSFRS-R is a validated and widely used instrument that assesses the fine and gross motor functions of the arms and legs, bulbar function and ventilation. It comprises 12 short questions with 5 anchor points $(0-4)$ as response options. The scale spans 0 to 48 points in total with the bottom end of the scale reflecting low functionality and more pronounced disease severity. A monthly decline in ALSFRS-R points, or delta ALSFRS-R, is indicative of the rate of deterioration and has prognostic significance (18).

\section{Self-assessment of symptoms of muscle stiffness and muscle weakness}

We intentionally used the terms "muscle stiffness" and "muscle weakness" to capture ALS symptoms as this reflected how participants evaluated their symptoms as they perceived them individually. What participants rated did not necessarily correspond to the clinical picture of spasticity and paresis. Arms 
and legs were assessed separately on a numeric rating scale (NRS) where a score of 0 signifies no stiffness/weakness while a score of 10 signifies most pronounced stiffness/weakness.

\section{Use of the MME}

The frequency of MME use was delineated as follows: once or twice a week, 3 to 4 times a week, 5 to 7 times a week, 8 to 10 times a week and more than 10 times a week.

\section{Recommendation of the MME}

The Net Promoter Score (NPS) was the instrument used to evaluate the participants' attitude towards the MME and their overall recommendation rates $(19,20)$. Originally, a tool created in customer relations management, the NPS has since been explored further and applied as an evaluation instrument in various clinical settings $(16,21-23)$. Adapted to our study objective, the NPS was calculated based on responses to the question: "How likely is it that you would recommend the MME to another friend or patient who is affected with ALS?"

Based on an 11-point Likert scale from 0 (recommendation absolutely unlikely) to 10 (recommendation highly likely) points, participants who gave the MME a 9 or a 10 were considered "promoters" (likely to recommend), those who gave it 7 or 8 points were considered "indifferent" and responders who gave the MME 6 to 0 points were considered "detractors" (unlikely to recommend). Finally, the percentage of detractors was subtracted from the promoters to ascertain the final score. In general, a group with a positive NPS is regarded as supportive and results over 50 are considered excellent (20). To avoid categorisation difficulties, an alternative is to omit the NPS calculus and merely report the average value that corresponds to the recommendation (19).

For the purpose of this study, we analysed the NPS for the total of participants as well as for individual groups according to gender, age, disease severity and frequency of use.

\section{Domain-oriented participants' experience with the MME}

To capture a participant's subjective impact of the MME on the various domains under treatment, an expert team of ALS neurologists and scientists with expertise in ALS care and observational studies developed statements around nine items. Based on our clinical experience the wording was aligned so as to reflect how a patient might perceive the changes that may occur in ALS. Therefore, we deliberately avoided medical-scientific terms such as spasticity, contractions or joint locking. The domains thus specified were: 'preservation of muscle strength', 'improvement of muscle strength', 'reduction of muscle stiffness', 'reduction of tendon shortening', 'diminution of the feeling of having rigid limbs', 'diminution of the feeling of being immobile', 'amplification of a sense of achievement', 'improvement of sleep quality' and 'improvement of general wellbeing'. The question we asked the patients was, "How do you rate the benefits you personally gained with the MME? Please specify for each domain." Participants could express their agreement or disagreement by giving each statement between 0 (no impact) and 10 points (best possible impact) on a Likert-scale. 


\section{Statistical methods}

Data were analysed with IBM SPSS Statistics (Version 25.0). Descriptive analyses were performed to compare frequencies within the parameters assessed. Results were expressed as means ( $\pm S D)$ if distribution was normal and as medians if numerical data were visualized or if distribution was nonGaussian. Significant differences between the parameters and, respectively, subgroups of nominally scaled data were assessed by applying contingency tables and Chi-square test. A statistically significant difference of paired samples was analysed by a t-test. The Wilcoxon test was employed for the analysis of statistical power of ordinally-scaled data, while metric data were subjected to the t-test. Correlational analysis was performed with Spearman's Rho because of the ordinal nature of the scales. For the comparison of the parameters weakness and stiffness, a ratio of both was formed for each participant. For group differences of nonparametric data, the Mann-Whitney Utest was performed for two independent samples and the Kruskal-Wallis one-way analysis of variance for independent samples of three or more. Statistical significance was ascertained according to an error risk of up to $5 \%$ (p-value < 0.05).

\section{Results}

\section{Sample characteristics}

Within the observation period, APST case managers initiated the provision of an MME to 237 patients, all of whom were subsequently invited to participate in the study. Ultimately, the respective study centres enrolled 144 patients in total.

\section{Demographic data and clinical characteristics}

The gender distribution (male:female) was 1.5:1, which is a common distribution in ALS $(24,25)$. The age of ALS onset was within the general range of prevalence in the ALS population (59.9 years SD 11.5) (26). At the time of initial MME use, the average disease course was 37.3 months (3.1 years). The survey was conducted after an average usage time of 8.2 months (SD 10.9), at which point in time the degree of impairment was severe at 27.2 ALSFRS-R points (SD 9.8) and disease progression was equivalent to a loss of 0.7 points per month.

Self-assessment revealed that $83.7 \%$ of participants experienced muscle stiffness in both the legs and arms and almost all (97.1\%) experienced weakness of the extremities. The results showed that both muscle stiffness and weakness were more pronounced in the legs. Only $17 \%$ of all patients reported exclusively either one of these symptoms.

In total, muscle weakness was significantly higher than muscle stiffness $(p<0.001)$. Weakness correlated with disease severity, as measured by the ALSFRS-R $(p<0.001$; correlation coefficient $=0.4)$ whereas no such correlation could be established with regard to muscle stiffness. Further information on demographic and clinical characteristics are presented in Table 1. 
Demographic and clinical characteristics of the cohort

\begin{tabular}{|c|c|c|}
\hline Characteristics & Classification & $\begin{array}{l}\text { Total cohort, } \\
\mathrm{n}=144\end{array}$ \\
\hline \multirow[t]{2}{*}{ Sex } & female, \% (n) & $36.8(53)$ \\
\hline & male, \% (n) & $63.2(91)$ \\
\hline \multirow[t]{2}{*}{ Age } & at onset, years, mean (SD, R) & $\begin{array}{l}59.9(11.5 ; 25.8- \\
81.5)\end{array}$ \\
\hline & at time of use of MMEt, years, mean (SD, R) & $\begin{array}{l}62.1(11.1 ; 29.6- \\
82.8)\end{array}$ \\
\hline Disease duration & $\begin{array}{l}\text { at time of use of MMEt, months, mean (SD, } \\
\text { R) }\end{array}$ & $\begin{array}{l}37.3(49.7 ; 3.0- \\
513.0)\end{array}$ \\
\hline Disease progression & mean $(S D, R)$ & $0.7(0.6 ; 0.0-2.8)$ \\
\hline ALS-FRS-R score (max. 48) & at time of survey, mean $(S D, R)$ & $27.2(9.8,1.0-40.0)$ \\
\hline \multirow[t]{3}{*}{ Presence of muscle stiffness } & total, yes, \% (n) & $83.7(113)$ \\
\hline & in lower extremities, \% (n) & $73.5(97)$ \\
\hline & in upper extremities, \% (n) & $69.6(94)$ \\
\hline \multirow[t]{3}{*}{ Severity of muscle stiffnessł } & mean $(S D, R)$ & $3.7(2.7,0-9)$ \\
\hline & in lower extremities, mean (SD, R) & $4.0(3.2 ; 0-10)$ \\
\hline & in upper extremities, mean (SD, R) & $3.4(3.1 ; 0-10)$ \\
\hline \multirow{3}{*}{$\begin{array}{l}\text { Presence of muscle } \\
\text { weakness }\end{array}$} & yes, \% (n) & $97.1(133)$ \\
\hline & in lower extremities, \% (n) & $94.1(127)$ \\
\hline & in upper extremities, \% (n) & $91.8(123)$ \\
\hline \multirow[t]{3}{*}{$\begin{array}{l}\text { Severity of muscle } \\
\text { weakness } \neq\end{array}$} & Total, mean (SD, R) & $4.6(2.3 ; 0-10)$ \\
\hline & in lower extremities, mean (SD, R) & $4.8(2.8 ; 0-10)$ \\
\hline & in upper extremities, mean (SD, R) & $4.3(2.8 ; 0-10)$ \\
\hline
\end{tabular}

Abbreviations: $n$, number of participants; SD, standard deviation; R, range; ALS-FRS-R, Amyotrophic Lateral Sclerosis Functional Rating Scale, revised; MME, Motor-assisted movement exerciser.

† Disease duration at the time of the initial use of the MME.

¥ Severity of muscle stiffness/weakness was assessed by using the numeric rating scale (NRS) with the allocation 0 meaning no stiffness/weakness to 10 meaning strongest stiffness/weakness. 


\section{Use of motor-assisted movement exerciser (MME)}

At the time of conducting the survey, the majority of participants $(n=138)$ were exercising on the MME at least 5 times a week $(59.4 \%, n=82)$. Still, $17.4 \%$ of participants surveyed $(n=24)$ were using the MME more than once a day on some days ( $\geq 8$ times per week; Figure 1).

Only two participants (1.4\%) had not yet started using the MME and another two participants $(1.4 \%)$ were no longer been able to use it due to disease progression.

\section{MME recommendation}

$71.6 \%$ of the responders $(n=101)$ strongly recommended the MME ( 9 to 10 points). $17.7 \%(n=25)$ were indifferent ( 7 to 8 points) and $10.6 \%(n=15)$ did not issue a recommendation ( 0 to 6 points). The NPS was +61 (Figure 2).

A further analysis of the frequency of MME use showed that participants who used the device at least 5 days a week ( 5 to 7 times a week) and several times a day ( $>7$ times a week; Figure 3 ) on some days were more likely to recommend the exerciser than the others.

Interestingly, recommendation rates did not differ significantly among participants who reported either greater stiffness or greater weakness of the extremeties. Women were more likely to recommend the MME than men (NPS: 67 vs. $58, p=0.019$ ). Participants over 60 years of age reached a higher NPS than younger responders, however, this finding was not of statistical significance (NPS: 65.5 vs. 54, $p=0.58$ ). Disease severity, as measured by the ALSFRS-R, did not have a significant impact on MME recommendation rates (ALSFRS-R groups subdivided by mean value: $\leq 27$ vs. $>27$ : NPS 68 vs. 59; $p=$ $0.531)$.

\section{Domain-oriented participant experience}

The following percentages depict the highest impact on specific treatment areas as described by participants and as reflected by a score of 7 to 10 (Figure 4; (in descending order): amplification of a sense of achievement $(67 \%)$, diminution of the feeling of having rigid limbs $(63 \%)$, diminution of the feeling of being immobile (61\%), improvement of general wellbeing (55\%), reduction of muscle stiffness (52\%), improved muscle flexing ability through stretching of tendons (45\%), preservation of muscle strength (39\%), improvement of sleep quality (27\%) and improvement of muscle strength $(20 \%)$.

There was a correlation between the reported overall benefit of MME treatment and frequency of MME use. It was highest among participants with more than 7 exercise sessions a week. A significant correlation was found between frequency of use and the domains "reduction of muscle stiffness", "diminution of the feeling of having rigid limbs", "diminution of the feeling of being immobile" and "improvement of general wellbeing" (Figure 5).

Participants with severely stiff limbs did not express a preference for specific MME characteristics. Participants with higher self-rated muscle weakness were significantly more likely to see the beneficial 
effects of the MME on the preservation and improvement of muscle strength $(p<0.05)$. The different age and gender groups reported similar experiences in all domains.

Although participants with greater weakness of the extremities gave higher ratings for overall improvement of muscle strength with the $\mathrm{MME}$, this applied predominantly to the earlier stages of the disease as measured by the ALSFRS-R $(p<0.05)$.

\section{Discussion}

\section{Sample selection, demographic and clinical characteristics}

The motor-assisted movement exerciser (MME) facilitates device-assisted physical therapy in a home setting to treat motor symptoms of the extremities in people with ALS and other neurological diseases.

At 144 participants with a confirmed diagnosis of ALS, this study presents the largest cohort to date in the investigation of user satisfaction with MME treatment and therapeutic efficacy. This substantial number is representative of the general ALS population in terms of gender distribution, age at onset and disease progression rate. Furthermore, the multicentre study design reflects the broad base across which the digitally supported APST care network operates. The selection criteria for participants included the medical indication for an MME and involvement in the care network, an offer that is primarily available at specialised ALS centres.

At 37 months at initiation of treatment, the mean ALS disease duration was notably long considering an average survival time of 3 to 4 years after symptom onset $(25,26)$. This may explain the high level of disease severity at the time of conducting the survey, i.e. another 8 months after initial MME treatment. This, of course, raises the question of what would be the ideal point in time for issuing a prescription for the device and of when and how the provision process ought to be started. As found in a previous study, provision failure rates are high (47\%) and, at 110 days, the latency between the establishment of the medical indication for the assistive device and its actual provision is remarkably high (2). There are several barriers to an early initiation of therapy, such as delayed determination of the indication or a timeconsuming intensive payer-side review of the indication prior to reimbursement. As one of the guiding objectives in the treatment of ALS is the preservation of functionality, exerciser-based treatment should be considered right at the initial onset of symptoms in the affected extremity to counter the detrimental effects of the disease. Our clinical experience supports this assumption but we will need to conduct further studies, preferably prospective in design, to provide conclusive clarification.

\section{Self-assessment of symptoms and domains}

We would like to stress that the self-reporting of symptom severity and prevalence was deliberately subjective by design.

Clinical experience shows that individuals that are affected but have no spasticity also report muscle stiffness, while the predominant presence of spasticity may also be perceived as weakness. 
Overall, muscle weakness was reported to be more severe and more frequent than muscle stiffness ( $97 \%$ vs. $84 \%$ ), and manifestations were more pronounced in the legs than in the arms. $83 \%$ of participants reported presence of both symptoms. Interestingly, muscle weakness correlated with both disease severity and progression rate whereas no such correlation could be established for muscle stiffness. Although naturally, the self-reporting format of motor symptoms is as such subjective in nature, our observations demonstrate the differential significance of weakness and stiffness to disease progression.

\section{Characteristics of MME use and use frequency}

The MME provides patients with high-frequency, motor-assisted physical therapy in the comfort of their own homes as an adjunct to physical therapy. It would be of interest to find out if access to standard physical therapy was more difficult to obtain for patients who were using the MME at a high frequency. Especially in regions or at times with limited access to standard physiotherapy (e.g. during the COVID-19 pandemic), regular sessions with the MME can compensate for a lack of general exercise.

In our study, MME use was characteristically extensive, as the majority of participants had at least five weekly exercise sessions. There was no correlation between the frequency of this routine and symptom severity or ALS disease progression as measured by the ALSFRS-R. The participants did not specify all by themselves how often they would use the MME. Each patient had been given an individual recommendation by one of their attending therapists well before the device was provided. Further adjustments were made over the course of treatment. These recommendations were issued independently of the present observational study and were based on the individual user's abilities and aims. At any time, the participants were free to adapt both frequency and intensity to their specific conditions. However, the sheer number of exercise sessions offers ample evidence of the good reception this device has received.

\section{Treatment recommendation and perception of MME therapy}

The domain-based user assessment showed a moderate benefit on the preservation of muscle strength. By comparison, participants gave higher ratings for the positive effect on stiffness. The domain of "stiffness" included the item of 'improved muscle flexing ability through stretching of tendons".

Substantially reduced muscle flexing ability is a relevant problem in ALS as this leads to contractures and can limit a patient's range of movement even further.

Participants most often reported that the sensation of being immobile and having rigid limbs was diminished and that they enjoyed a sense of accomplishment and improved general wellbeing. Those participants who highlighted that their muscles were less stiff, that the feeling of having rigid limbs or being immobile was diminished and that they felt better in general were the ones that used the MME more often than others. The likelihood of perceiving a beneficial effect of MME use on the preservation and improvement of muscle strength was higher when participants rated their muscle weakness as more severe and were in the earlier stages of the disease. In view of the fact that most participants were strong 
recommenders, although women and older participants more so, we come to the conclusion that the benefits of use are not particular to one group or another, but rather a highly individual matter in the ALS cohort. In this regard, separate assignment of MME treatment to muscle stiffness or weakness does not appear reasonable.

\section{Limitations}

The present study results rely on the analysis of the participants' subjective responses. Patient selection was based on the individual indication for MME treatment, actual supply and willingness to participate in the study. While this is implicative of a selection bias and constitutes a limitation of this study, the sample size is such that the actual impact is not all that strong.

With regard to explicitness of results, we must certainly differentiate between evaluations performed by patients and those by distinguished medical professionals. However, it was the very purpose of this study to focus on the subjectivity of treatment effects, i.e. on the fine interplay between the issue of recommendations for device-based training and the personal experience and opinion of the patients themselves.

Although definitive medical criteria for the diagnosis of ALS were applied in this study, the system according to which symptoms were reported has not been validated. Therefore, all we were left with was to analyse the patients' subjective stance on the effectiveness of specific symptoms, i.e. the user perspective. We will require further studies to define the medically measurable effects of MME use on specific functionalities.

\section{Conclusion}

This study demonstrates the high value that patients report deriving from the MME. Notwithstanding, the distinction between muscle weakness and stiffness remains equivocal. The positive effects are described separately for either cluster of symptoms; however, in reality, these can hardly be regarded separately. One of the key characteristics of the MME is the integration of active and passive exercise, i.e. it allows for motor-assisted and passive limb movement as well as for active exercise. Due to the severity and progress of symptoms in ALS, most of the participants in this study would likely not have been able to pursue the active exercise regime only. In view of this fact, the MME gains in both value and importance in the range of therapeutic and assistive devices currently available to patients. It has the capacity to play a crucial supportive role in the realm of neurological diseases such as ALS.

In conclusion, the beneficial effects are perceptible beyond a merely functional level. The findings of enhancement of wellbeing and resolve of perceived inactivity are consistent with reports from a previous study (27).

Although the MME is a device that is indicated medically and has received positive user feedback, it is frequently not supplied to patients. As for now, we can only speculate what this may be due to. To 
elucidate potential obstacles to early therapy implementation and a consistent provision process, further studies are needed.

\section{Declarations}

\section{Availability of data and materials}

The data that support the findings of this study are available from Ambulanzpartner Soziotechnologie APST GmbH but restrictions apply to the availability of these data, which were used under license for the current study, and so are not publicly available. Data are however available from the authors upon reasonable request and with permission of Ambulanzpartner Soziotechnologie APST GmbH.

\section{Competing interests}

AM received presentation and consulting fees from Merz Pharma GmbH \& Co. KGaA and ITF Pharma $\mathrm{GmbH}$. TM received consulting fees from Cytokinetics, GSK and Desitin Arzneimittel GmbH; served on scientific advisory boards for Cytokinetics, GSK and TEVA. TM and CM are founders of the internet platform Ambulanzpartner and hold shares of Ambulanzpartner Soziotechnologie APST GmbH. All other authors declare that they have no conflicts of interest.

\section{Funding}

This study was supported by a research grant from medica Medizintechnik GmbH to APST. Sponsors were not involved in the study design, collection, analysis, and interpretation of data, in the writing of the report, or in the decision to submit the article for publication.

\section{Authors' contributions}

AM wrote the manuscript, planned the study, performed statistical analyses, interpreted the data, and was responsible for on-site participant enrolment and data collection. SS and TM planned the study, performed statistical analyses, interpreted the data, coordinated the cooperation between the centres involved, and critically revised the manuscript. $\mathrm{MG}, \mathrm{BW}, \mathrm{CM}$, and $\mathrm{BH}$ were responsible for local data collection and contribution, coordinated the cooperation between the centres involved, implemented the study at the centres, and were responsible for database and documentation integrity. TG, UW, RS, JG, AR, JCK, TL, PW, RG, JW, PB, MM, JD, ACL, DK, JN, RYK were responsible for on-site study implementation, participant enrolment, data collection, and critically revised the manuscript.

\section{Acknowledegements}


The authors wish to thank all patients for participating in this study.

\section{References}

1. Statland JM, Barohn RJ, McVey AL, Katz JS, Dimachkie MM. Patterns of Weakness, Classification of Motor Neuron Disease, and Clinical Diagnosis of Sporadic Amyotrophic Lateral Sclerosis. Neurologic Clinics. 2015;33(4):735-48.

2. Funke A, Spittel S, Grehl T, Grosskreutz J, Kettemann D, Petri S, et al. Provision of assistive technology devices among people with ALS in Germany: a platform-case management approach. Amyotrophic Lateral Sclerosis and Frontotemporal Degeneration. 2018;19(5-6):342-50.

3. Ortega-Hombrados L, Molina-Torres G, Galán-Mercant A, Sánchez-Guerrero E, González-Sánchez M, Ruiz-Muñoz M. Systematic Review of Therapeutic Physical Exercise in Patients with Amyotrophic Lateral Sclerosis over Time. International Journal of Environmental Research and Public Health. 2021;18(3).

4. Dal Bello-Haas V. Physical therapy for individuals with amyotrophic lateral sclerosis: current insights. Degenerative Neurological and Neuromuscular Disease. 2018;Volume 8:45-54.

5. Deforges S, Branchu J, Biondi O, Grondard C, Pariset C, Lécolle S, et al. Motoneuron survival is promoted by specific exercise in a mouse model of amyotrophic lateral sclerosis. The Journal of Physiology. 2009;587(14):3561-72.

6. Kalron A, Mahameed I, Weiss I, Rosengarten D, Balmor GR, Heching M, et al. Effects of a 12-week combined aerobic and strength training program in ambulatory patients with amyotrophic lateral sclerosis: a randomized controlled trial. Journal of Neurology. 2021;268(5):1857-66.

7. Barclay A, Paul L, MacFarlane N, McFadyen AK. The effect of cycling using active-passive trainers on spasticity, cardiovascular fitness, function and quality of life in people with moderate to severe Multiple Sclerosis (MS); a feasibility study. Multiple Sclerosis and Related Disorders. 2019;34:12834.

8. Diserens K, Perret N, Chatelain S, Bashir S, Ruegg D, Vuadens P, et al. The effect of repetitive arm cycling on post stroke spasticity and motor control. Journal of the Neurological Sciences. 2007;253(1-2):18-24.

9. Ridgel AL, Phillips RS, Walter BL, Discenzo FM, Loparo KA. Dynamic High-Cadence Cycling Improves Motor Symptoms in Parkinson's Disease. Frontiers in Neurology. 2015;6.

10. Nardone R, Orioli A, Golaszewski S, Brigo F, Sebastianelli L, Höller Y, et al. Passive cycling in neurorehabilitation after spinal cord injury: A review. The Journal of Spinal Cord Medicine. 2016;40(1):8-16.

11. Nardone R, Langthaler PB, Bathke AC, Höller Y, Brigo F, Lochner P, et al. Effects of passive pedaling exercise on the intracortical inhibition in subjects with spinal cord injury. Brain Research Bulletin. 2016;124:144-9. 
12. Vandenbroucke JP, von Elm E, Altman DG, Gøtzsche PC, Mulrow CD, Pocock SJ, et al. Strengthening the Reporting of Observational Studies in Epidemiology (STROBE): Explanation and Elaboration. PLoS Medicine. 2007;4(10).

13. Brooks BR, Miller RG, Swash M, Munsat TL. El Escorial revisited: Revised criteria for the diagnosis of amyotrophic lateral sclerosis. Amyotrophic Lateral Sclerosis and Other Motor Neuron Disorders. 2009;1(5):293-9.

14. Fürstenau D, Klein S, Vogel A, Auschra C. Multi-sided platform and data-driven care research. Electronic Markets. 2021.

15. Meyer T, Kettemann D, Maier A, Grehl T, Weyen U, Grosskreutz J, et al. Symptomatic pharmacotherapy in ALS: data analysis from a platform-based medication management programme. Journal of Neurology, Neurosurgery \& Psychiatry. 2020;91(7):783-5.

16. Meyer R, Spittel S, Steinfurth L, Funke A, Kettemann D, Münch C, et al. Patient-Reported Outcome of Physical Therapy in Amyotrophic Lateral Sclerosis: Observational Online Study. JMIR Rehabilitation and Assistive Technologies. 2018;5(2).

17. Maier A, Holm T, Wicks P, Steinfurth L, Linke P, Münch C, et al. Online assessment of ALS functional rating scale compares well to in-clinic evaluation: A prospective trial. Amyotrophic Lateral Sclerosis. 2012;13(2):210-6.

18. Kimura F, Fujimura C, Ishida S, Nakajima H, Furutama D, Uehara H, et al. Progression rate of ALSFRS$R$ at time of diagnosis predicts survival time in ALS. Neurology. 2006;66(2):265-7.

19. Krol MW, de Boer D, Delnoij DM, Rademakers JJDJM. The Net Promoter Score - an asset to patient experience surveys? Health Expectations. 2015;18(6):3099-109.

20. Reichheld F. The ultimate question 2.0 (revised and expanded edition): How net promoter companies thrive in a customer-driven world: Harvard Business Review Press; 2011.

21. Stein N, Brooks K. A Fully Automated Conversational Artificial Intelligence for Weight Loss: Longitudinal Observational Study Among Overweight and Obese Adults. JMIR Diabetes. 2017;2(2).

22. Meyer T, Maier A, Uzelac Z, Hagenacker T, Günther R, Schreiber-Katz O, et al. Treatment expectations and perception of therapy in adult patients with spinal muscular atrophy receiving nusinersen. European Journal of Neurology. 2021.

23. Wilberforce $M$, Poll S, Langham $H$, Worden A, Challis D. Measuring the patient experience in community mental health services for older people: A study of the Net Promoter Score using the Friends and Family Test in England. International Journal of Geriatric Psychiatry. 2018;34(1):31-7.

24. Rosenbohm A, Liu M, Nagel G, Peter RS, Cui B, Li X, et al. Phenotypic differences of amyotrophic lateral sclerosis (ALS) in China and Germany. Journal of Neurology. 2018;265(4):774-82.

25. Spittel S, Maier A, Kettemann D, Walter B, Koch B, Krause K, et al. Non-invasive and tracheostomy invasive ventilation in amyotrophic lateral sclerosis: Utilization and survival rates in a cohort study over 12 years in Germany. European Journal of Neurology. 2020;28(4):1160-71.

26. Talbott EO, Malek AM, Lacomis D. The epidemiology of amyotrophic lateral sclerosis. Neuroepidemiology. Handbook of Clinical Neurology2016. p. 225-38. 
27. Bond Jr V, Retta T, Kumar K, Dorsey J, Gorantla VR, Millis RM. Mood Responses to Passive and Active Motion Leg Cycling Exercise in Healthy Sedentary Young Adults. Advances in Preventive Medicine. 2020;2020:1-7.

\section{Figures}

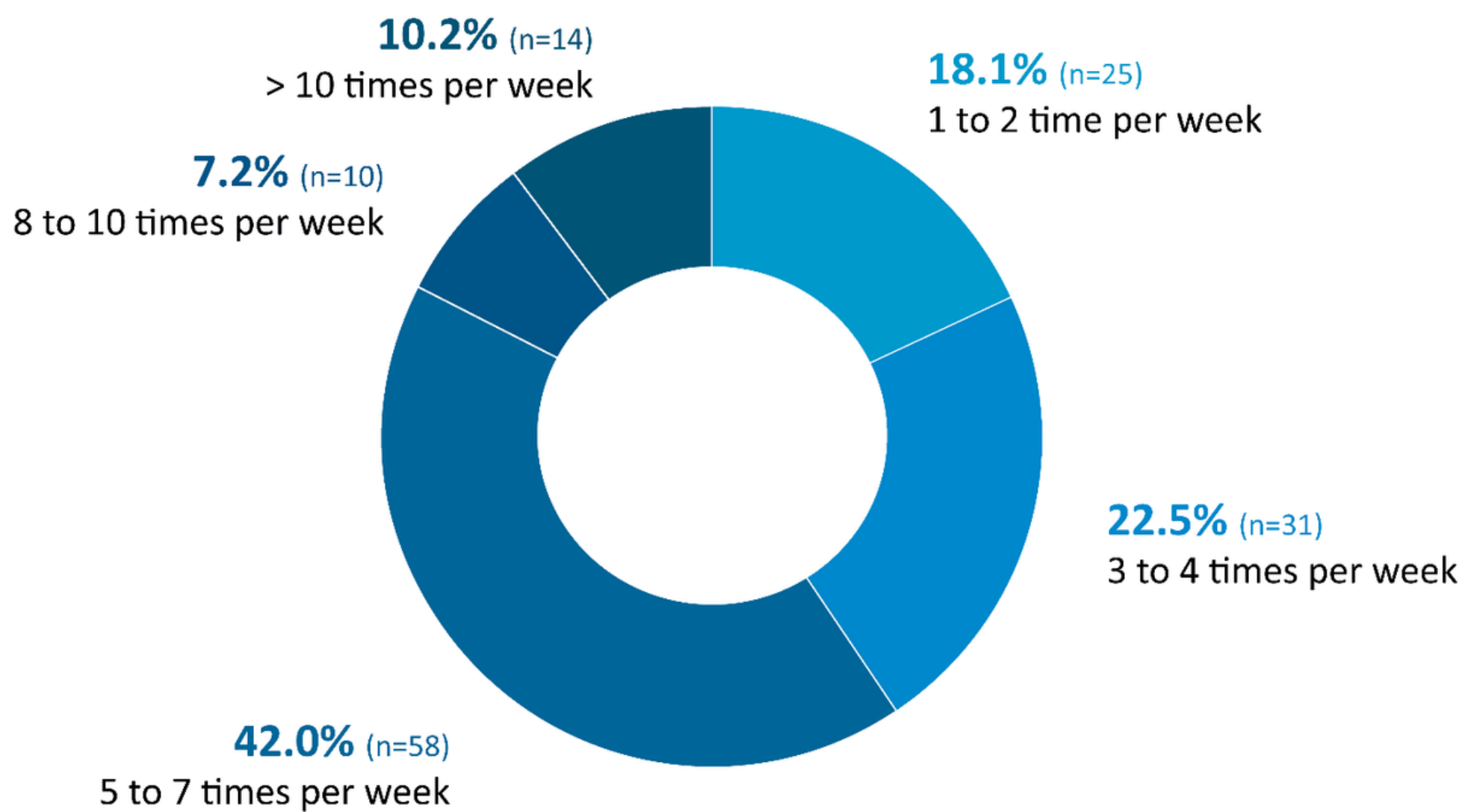

\section{Figure 1}

Use of motor-assisted movement exerciser (MME). 
A)

Likely

recommendation

Indifferent

recommendation

Unlikely

recommendation

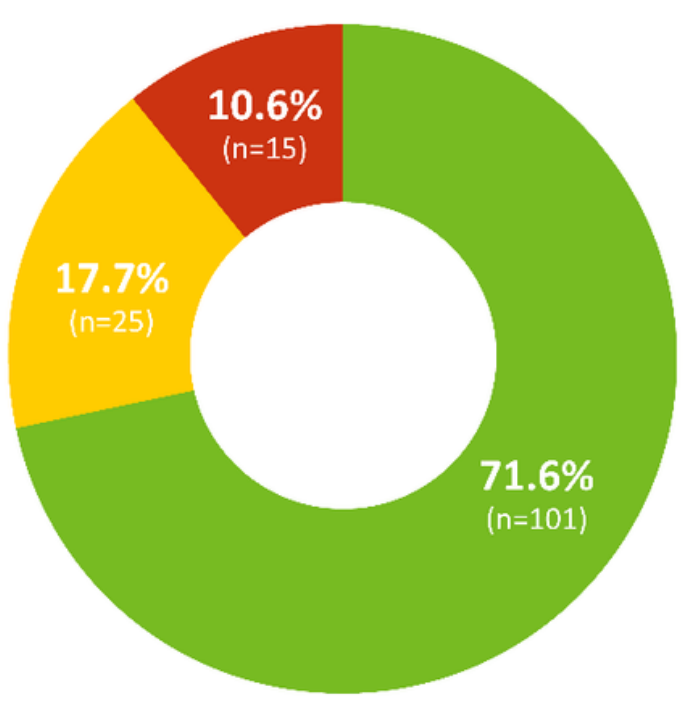

B)

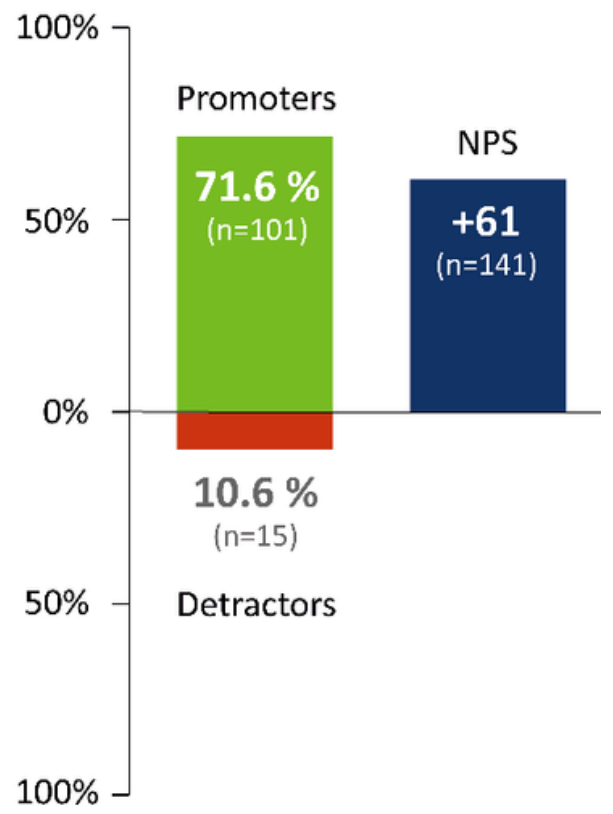

Figure 2

Recommendation of motor-assisted movement exerciser (MME).

\section{Figure 3}

Recommendation of motor-assisted movement exerciser (MME) with respect to frequency of MME use. 


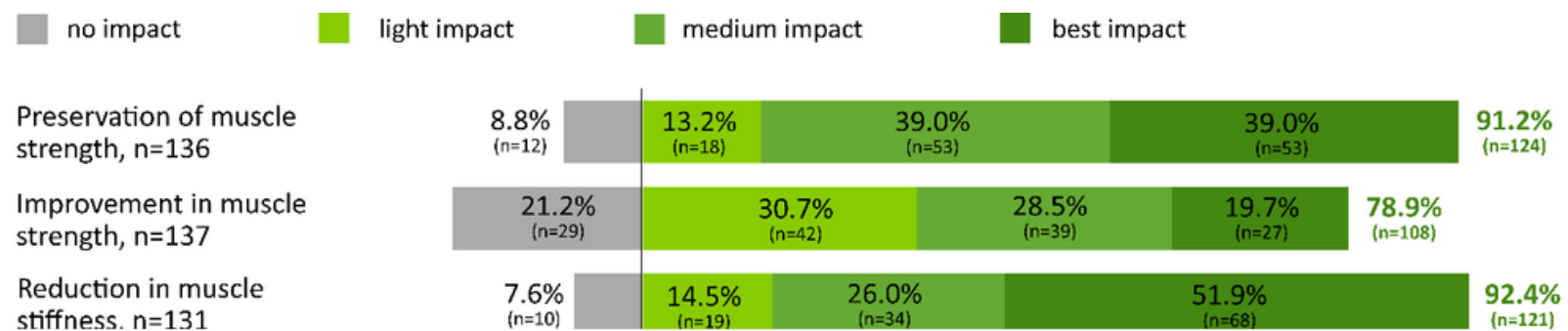

\section{Figure 4}

Domain-orientated participant experiences of the MME.

\section{Figure 5}

Domain-orientated participant experiences with respect to frequency of MME. 